\title{
Incidental demyelinating inflammatory lesions in asymptomatic patients: a Brazilian cohort with radiologically isolated syndrome and a critical review of current literature
}

\author{
Desmielinização inflamatória incidental em indivíduos assintomáticos: uma coorte \\ brasileira com síndrome radiológica isolada e uma revisão crítica da literatura atual \\ Antonio Carlos Martins Maia Jr.1, Antonio José da Rocha¹, Bernardo Rodi Barros², Charles Peter Tilbery³
}

\begin{abstract}
Despite the definition of specific diagnostic criteria to identify radiologically isolated syndrome (RIS) suggestive of multiple sclerosis, its natural history remains incompletely understood. We retrospectively analyzed a Brazilian cohort of 12 patients to clarify their features and to emphasize the role of imaging predictors in clinical conversion. We demonstrated that, although some individuals did not exhibit progression over a lengthy follow-up period (16.7\%), most patients will progress clinically or radiologically in the initial years of the follow-up (83.3\%). Infratentorial and spinal cord involvement, as well as the total number of lesions, were more relevant predictors of progression than gadolinium enhancement. Further studies remain necessary to define the risk of conversion in males and to clarify the cognitive abilities of RIS patients. This study may provide an improved understanding of the natural course and evolution of incidental magnetic resonance imaging lesions, and further assists with the management of RIS in clinical practice.
\end{abstract}

Key words: multiple sclerosis, radiologically isolated syndrome, incidental demyelination, magnetic resonance.

\section{RESUMO}

Apesar da definição dos critérios diagnósticos específicos para identificar a síndrome radiológica isolada (SRI) sugestiva de esclerose múltipla, sua história natural ainda não foi completamente entendida. Analisamos retrospectivamente uma coorte brasileira de 12 pacientes visando a esclarecer suas características e enfatizar o papel da imagem em predizer conversão clínica. Embora alguns indivíduos não apresentem progressão em longo período de acompanhamento (16,7\%), a maioria dos pacientes com SRI progrediu clínica ou radiologicamente nos primeiros anos do seguimento (83,3\%). 0 comprometimento infratentorial e da medula espinhal, bem como o número total de lesões, foram mais relevantes em predizer a progressão que a presença de impregnação pelo gadolínio. Estudos futuros são necessários para definir o risco de conversão em homens e para esclarecer a repercussão cognitiva da SRI. Este estudo pode ampliar o entendimento da história natural e da evolução das lesões incidentais à imagem de ressonância magnética contribuindo para a adequada condução clínica da SRI.

Palavras-Chave: esclerose múltipla, síndrome radiológica isolada, desmielinização incidental, ressonância magnética.

The term radiologically isolated syndrome (RIS) was recently introduced to describe patients who have incidental brain magnetic resonance imaging (MRI) abnormalities suggestive of multiple sclerosis (MS), but without any signs or symptoms attributable to the disease ${ }^{1}$. The proposed diagnostic criteria include brain MRI to establish anatomic dissemination in space (DIS) in the absence of a better explanation or a clinical history of inflammatory demyelinating disease of the central nervous system (CNS). RIS is well known in clinical practice; however, the natural history of this condition remains incompletely understood ${ }^{2}$.

The current literature has demonstrated that RIS patients may develop clinical symptoms, converting to either relapsingremitting or primary progressive $\mathrm{MS}^{3}$. Alternatively, patients may show progression in the MRI lesions without any objective clinical symptoms (radiologic progression); patients may even show

${ }^{1}$ MD, PhD Division of Neuroradiology, Fleury Medicina e Saúde, São Paulo SP, Brazil; Division of Neuroradiology, Santa Casa de Misericórdia de São Paulo, São Paulo SP, Brazil;

${ }^{2}$ MD, Division of Neuroradiology, Santa Casa de Misericórdia de São Paulo, São Paulo SP, Brazil;

${ }^{3} \mathrm{MD}$, PhD Division of Neurology, Santa Casa de Misericórdia de São Paulo, São Paulo SP,Brazil.

Correspondence: Antonio José da Rocha; Rua Dr. Cesário Motta Junior 112; 01221-020 São Paulo SP - Brasil; E-mail: a.rocha@uol.com.br

Conflict of interest: There is no conflict of interest to declare.

Received 26 May 2011; Received in final form 01 September 2011; Accepted 08 September 2011 
stabilized brain abnormalities in subsequent imaging examinations $^{1-6}$. The aim of this report is to describe retrospectively the clinical-radiological features and evolution of the first Brazilian series of patients with RIS. Clinical and imaging predictors were analyzed. Our data were also compared with previous findings.

\section{METHODS}

The protocol was approved by the Institutional Committee of Human Research, and informed consent was obtained from all patients. This is a retrospective analysis of clinicalradiological data from a series of patients diagnosed with RIS in Santa Casa de Misericórdia de São Paulo and Fleury Medicina e Saúde from January 2002 to March 2011. The diagnoses were defined in accordance with recently proposed criteria $^{1}$. Patients who underwent a clinical follow-up and at least two imaging examinations were included in this study. To confirm the diagnosis of RIS, we performed comprehensive neurological evaluations and detailed clinical histories, including an active search for symptoms attributable to MS. Differential diagnosis for multifocal T2 abnormalities was actively conducted according to current criteria?.

We selected patients that underwent comparable MRI protocols, including Axial FLAIR(TR/TE/TI 11000/140/2600 ms), T2-weighted spin echo (SE) (5000/101 ms) and T1-weighted $\mathrm{SE}$ with and without magnetization transfer pulse (TR/TE, 510/12; MT pulse) sequences. We also examined T1 sequences (SE and SE/MT) after intravenous gadolinium (Gd) administration. Spinal cord studies included T1- and T2-weighted SE sequences. T1 SE sequences with fat saturation after intravenous Gd administration were also analyzed.

According to established neurological criteria, clinical symptoms were considered in the appropriate clinical settings as clinically isolated syndrome (CIS) related to MS or as clinically definite MS (CDMS $)^{8}$. In the absence of any objective clinical symptoms, radiologic progression was defined as the presence of a new T2 focal abnormality, Gd enhancement or enlargement of pre-existing lesions in longitudinal follow-up scans. Imaging stability of the abnormalities was considered in subsequent examinations when neither clinical symptoms nor radiologic progression were documented. MRI abnormalities and follow-up assessments were analyzed collaboratively by the authors.

Tests for interaction were performed for age, gender, the presence of oligoclonal bands in cerebrospinal fluid (CSF), the number of lesions and the presence of Gd enhancement, infratentorial lesions or spinal cord lesions in the baseline MRI. These potential predictors of conversion to MS or of the development of new brain lesions were analyzed using the Cox regression model, generating hazard ratios (HR) with 95\% confidential intervals and $p$ values $<0.05$. The duration of the followup period was also confronted to each patient's outcomes.

\section{RESULTS}

Sixteen patients ( 14 females, 2 males) were diagnosed with RIS during the study period. According to our defined criteria, 4 patients (all female) were excluded from this study due to the availability of only one imaging examination. Table 1 summarizes the demographic, clinical, laboratory and imaging data from our series of the remaining 12 patients, as well as the evolution of these parameters. Patient ages ranged from 17 to 54 years, with a median age of 38.7 years (standard deviation of 9.5 years). The median follow-up period for these patients was 49.2 months (range 11-109 months). All patients had a normal neurological examination at the first MRI scan, without any familial history of MS.

Symptoms that led to the first MRI scan included primary headache (7/12-58.3\%) and depression unrelated to a typical inflammatory-demyelinating CNS syndrome (2/12-16.7\%). One patient reported hyperprolactinemia, while another patient had a pineal cyst previously observed on a computed tomography scan. One patient was being investigated for a long-lasting horizontal nystagmus (Table 2).

Brain MRI scans were available from all 12 patients; spinal cord studies (cervical segment at least) were only available for 5 of the patients. The baseline brain MRI scans exhibited DIS according to the Barkhof criteria9. We documented more than nine T2 hyperintensities (11/12-91.6\%), three or more periventricular T2-weighted lesions (11/12-91.6\%), infratentorial lesions (6/12-50\%), juxtacortical lesions (12/12$100 \%)$ and Gd enhancement (4/12-33.3\%).

Radiologic progression was documented in $50 \%$ of patients (6/12), while MRI stability without the development of any objective clinical symptoms was documented in $16.7 \%$ of patients from our series (Fig 1). Four patients (4/12-33.3\%) exhibited paresthesia attributable to a demyelinating event. Two of those patients (2/12-16.7\%) were diagnosed with CIS (mean follow-up period - 12 months), while the 2 remaining patients (2/12-16.7\%) had recurrent symptoms compatible with CDMS (mean follow-up period of 57.5 months).

In each patient, the total lesion number varied from 5 to 36 lesions (median=14; SD 9.4), with a periventricular distribution predominating. A statistically significant correlation was found between the total number of lesions in the first examination and subsequent clinical conversion $(\mathrm{p}=0.017)$. Patients in the subgroup that developed clinical manifestations during the follow-up period exhibited, on average, 26 focal lesions in the first MRI scan (Fig 2). In contrast, patients in the subgroup without symptoms exhibited only, on average, 12.3 lesions in the first scan. There was no statistically significant difference in the number of lesions predicting CIS or CDMS ( $p=0.65)$. Furthermore, 50\% (6/12) of our patients exhibited infratentorial lesions in the first examination. This parameter was also significant to predict clinical conversion $(\mathrm{p}=0.017)$. 

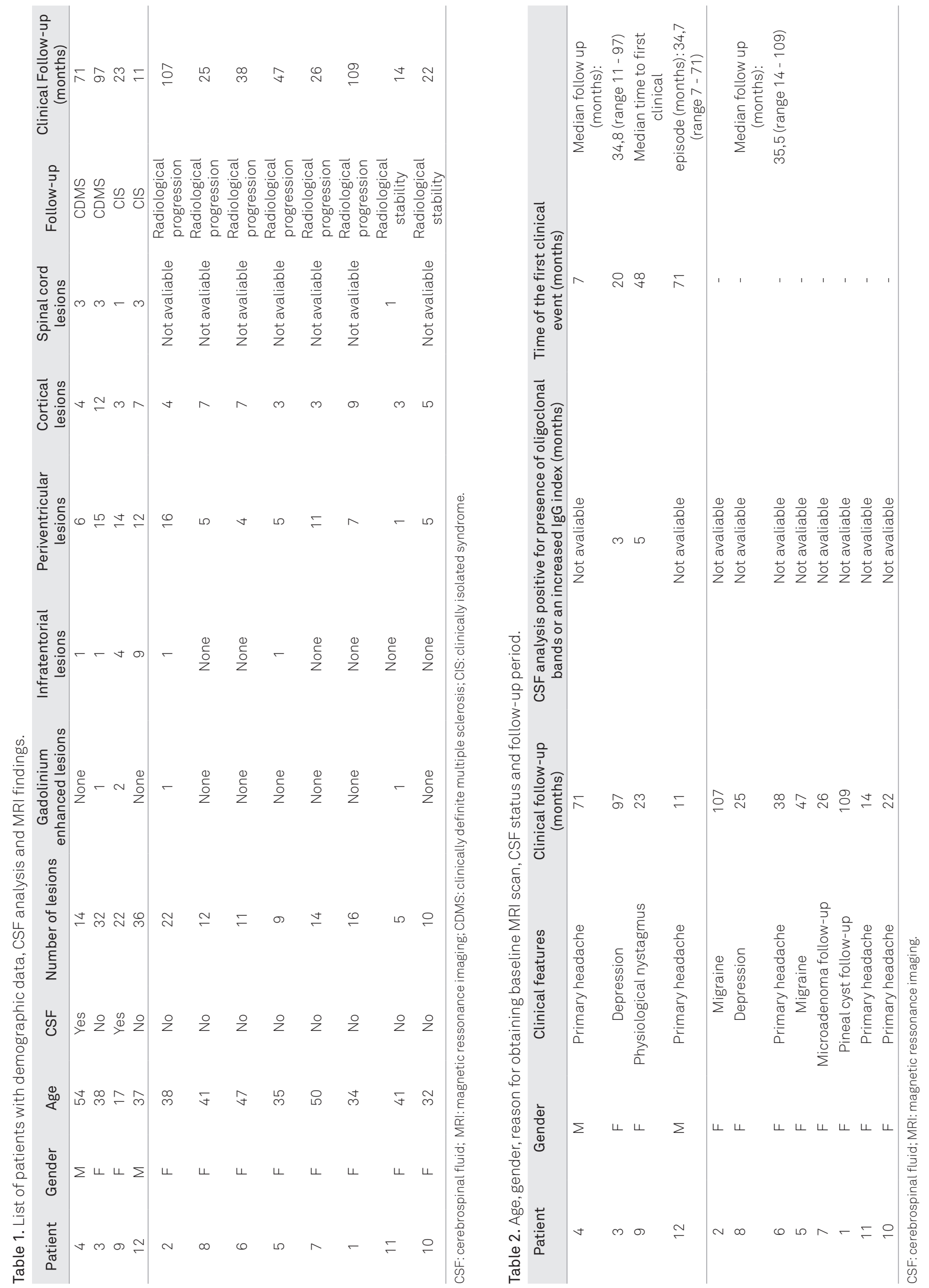


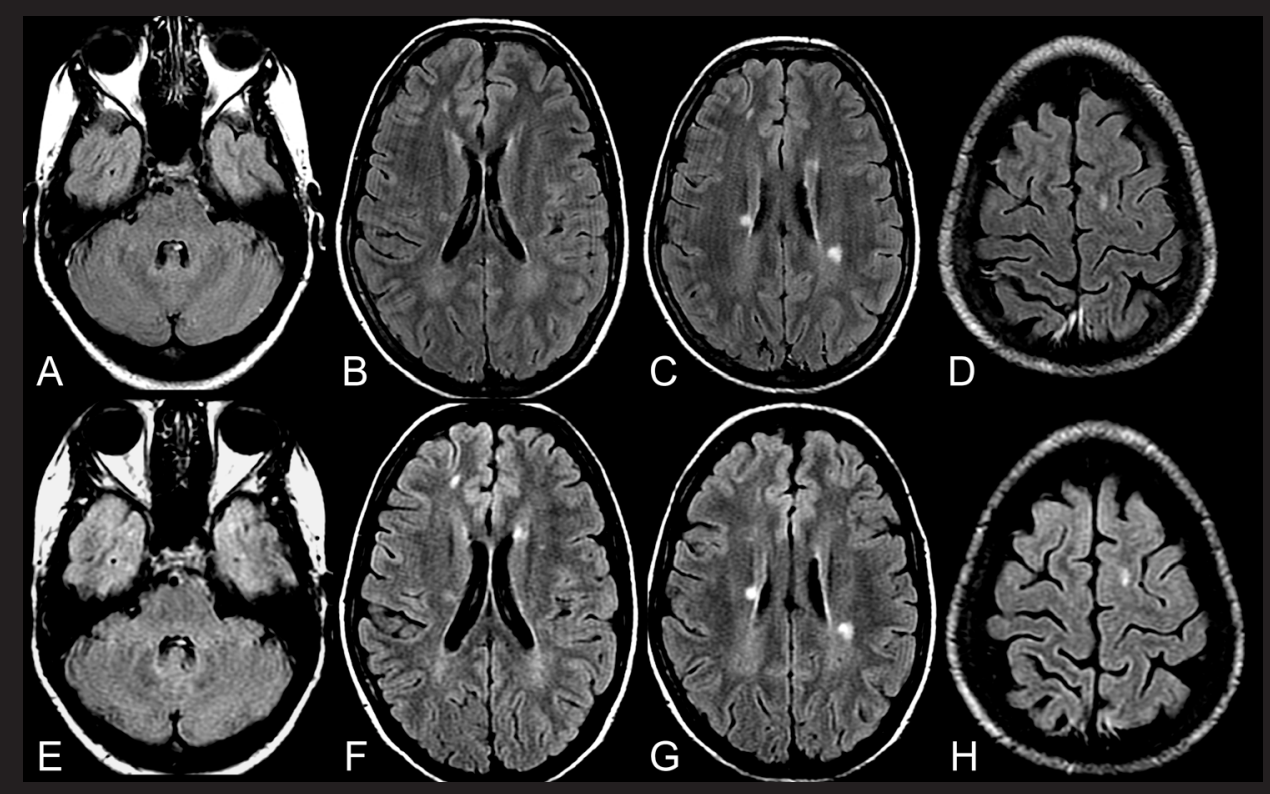

Fig 1. Radiologic stability (patient \#10). Axial FLAIR images at baseline (Jan-2009) (A-D) and follow-up (May-2010) (E-F). Note the presence of unchanged lesions in the periventricular white matter and at the juxtacortical region (total=10 lesions).

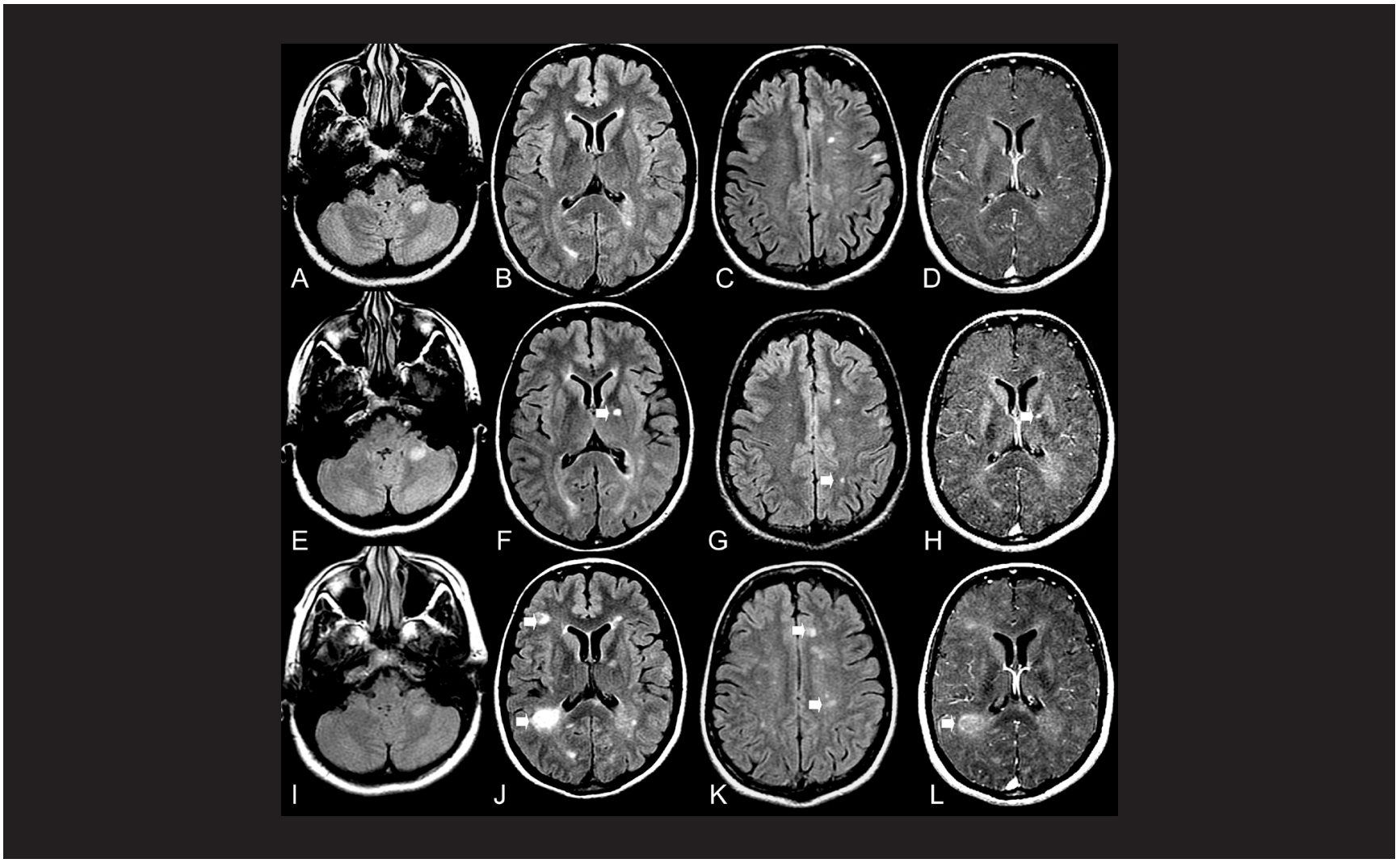

Fig 2. Radiologic progression and subsequent clinical conversion during the follow-up period (patient \#3). Baseline MRI (2003) - Axial FLAIR images (A-C) and axial T1 SE MT image after intravenous Gd administration. Note juxtacortical, periventricular and cerebellar lesions (total=32 lesions) with no evidence of blood-brain barrier disruption. Comparative images in 2007 (D-G) exhibited radiologic progression of lesions. Note the appearance of new lesions in FLAIR images not previously seen in the baseline study (arrows). Also note focal Gd enhancement, denoting acute inflammatory activity. At the time that these images were acquired, there were no clinical symptoms. A new comparative study was obtained in 2010 (H-K), exhibiting new lesions in FLAIR images and new foci of Gd enhancement. Clinical symptoms emerged during the last examination; this patient was considered clinically definite multiple sclerosis. 
Spinal cord lesions compatible with focal demyelination were demonstrated in all examined patients from our series (five patients). Clinical symptoms were documented in the follow-up of four patients from this subgroup. Focal inflammatory activity, characterized by Gd enhancement, was observed in the first examination in four patients from this cohort (4/12-33.3\%). No statistical correlation between Gd enhancement and clinical conversion was found $(\mathrm{p}=0.43)$.

Focal brain hypointensities on $\mathrm{T} 1$ sequences (i.e., black holes) consistent with irreversible damage were documented in two patients $(2 / 12-16.7 \%)$ in the first MRI scan. Clinically definite MS was confirmed in one of these patients. Two different patients $(2 / 12-16.7 \%)$ developed this pattern during the follow-up period. One patient from this subgroup converted to CDMS, and the other patient converted to radiologic progression.

\section{DISCUSSION}

Incidental demonstration of demyelinating lesions has been reported in autopsies and in familial and twin studies ${ }^{10-13}$. However, the advent of MRI has increased the possibility of demonstrating multifocal brain and spinal cord lesions compatible with MS, even in unsuspected scenarios $^{1-6,14}$. Postmortem studies from the pre-MRI era have revealed typical pathologic findings of MS in patients who were apparently asymptomatic during their lifetimes ${ }^{11}$; these studies have also reported unexpected lesions in some patients ${ }^{15}$.

With the extensive availability of brain and spinal cord MRI at present, imaging studies performed for indications other than suspicion of demyelinating disease may reveal lesions in patients that are highly suggestive of MS (i.e., with ovoid, well-circumscribed, homogeneous and periventricular distribution). In 2009, Okuda et al. ${ }^{1}$ proposed the concept of radiologically isolated syndrome to describe this scenario based on Barkhof's criteria ${ }^{9}$ to confirm DIS.

Structural neuroimaging studies of patients with an initial clinical event suggestive of MS usually reveal other brain or spinal lesions. Most of these CNS lesions present at the time of MS diagnosis were asymptomatic, suggesting the existence of a presymptomatic period of unknown duration. New asymptomatic lesions also often appeared during the course of established MS, confirming the subclinical activity of the disease $\mathrm{e}^{4,16,17}$.

Currently, there is neither a single clinical feature nor a sole diagnostic test that can confirm the diagnosis of MS. A recent revision of the International Panel Criteria confirmed MRI as the most effective paraclinical tool to define DIS dissemination in time (DIT) for the diagnosis of MS in patients with at least one clinical event consistent with a demyelinating disease after exclusion of alternative possibilities ${ }^{18}$. These criteria have been successful in defining the diagnosis of MS in the first clinical manifestation without compromising specificity and accuracy ${ }^{19-21}$. Using only a single MRI analysis, all patients in our study exhibited DIS, and $33.3 \%$ of the patients exhibited DIT according to these new criteria ${ }^{19,21}$. Furthermore, clinical conversion was only documented in $33.3 \%$ of all cases (2/12 of CIS; $2 / 12$ of CDMS). Our data suggest that all MRI criteria in RIS cases should be used parsimoniously as long as MS remains an exclusionary diagnosis. Unless patients develop a clinical symptom typical of a demyelinating episode, MRI analysis alone is not enough to confirm the diagnosis of $\mathrm{MS}^{1,2,22}$.

In our series of patients, there was no difference in median ages between both groups (36.75 and 39.75 years old with and without clinical symptoms, respectively). In our cohort, patients who presented CIS were younger on average (median 27.0 years old) than patients in the entire cohort (median 46 years old). In our study, patients with CDMS were not as young as those with CIS. In our review of the current literature, we found that all previously described studies reported the predominant occurrence of RIS in women ${ }^{1-6}$. Although RIS was more prevalent in women, we determined that in these previous studies the rate of conversion to CDMS was higher in males as compared to that of females ${ }^{1-4,6,23}$. Both men in our series converted to CDMS during the follow-up period. However, this tendency should be further confirmed in larger studies.

In this small cohort of patients with RIS, only two patients exhibited stabilized brain abnormalities and did not develop clinical symptoms consistent with MS during the follow-up period (mean duration of 18 months). Although the follow-up period in this study was relatively short, we emphasize that the number of lesions on the baseline MRI scans was smaller than that noted in remaining patients. It is important to note that a long-term follow-up period is mandatory to correctly define stabilized brain abnormalities.

Radiologic progression in our series of patients was in line with previous reports ${ }^{1-6,14}$. Radiologic progression occurred in $50 \%(6 / 12)$ of our patients, with a mean follow-up time of 2.8 years. Okuda et al. ${ }^{1}$ found a radiologic progression rate of $59 \%$, with a median follow-up time of 2.7 years. Our results showed a tendency toward earlier radiologic progression and clinical conversion to MS in proportion to the total basal number of lesions. This observation appears to be supported by the same arguments explaining the high risk of conversion following CIS with brain and spinal cord asymptomatic lesions ${ }^{14,17,24,25}$. Radiologic progression in RIS is in line with a recent observation in CIS cohorts that confirmed the occurrence of a long-term radiologic progression, albeit less commonly than in $\mathrm{CDMS}^{26}$.

There is much evidence supporting the importance of the preclinical phase of $\mathrm{MS}^{14}$. RIS is likely to be a heterogeneous syndrome rather than simply presymptomatic $\mathrm{MS}^{27}$. Patients may have a presymptomatic stage of MS with an expected clinical evolution to symptomatic MS; alternatively, patients may 
remain with only subclinical activity and no evolution to MS (i.e., radiologic progression). The impact of RIS on subsequent neurologic outcomes remains unclear, although it has been examined in the current literature ${ }^{2,27,28}$. Lebrun et al. ${ }^{23}$ recently suggested that RIS patients have similar cognitive profiles to MS patients. It could be suggested that RIS patients are MS patients with an undiagnosed, isolated symptom presenting as cognitive dysfunction. It is possible that there exists a destructive process that remains below the clinical threshold of detection (i.e., subclinical MS). Therefore, new MR techniques, including MT, spectroscopy or diffusion tensor imaging might clarify the relevance of preclinical lesions and thus improve our understanding of unexpected manifestations in further analyses.

In contrast with previous studies, Gd enhancement was not confirmed as a predictor of either radiologic or clinical progression $^{1,3}$. It is possible that our results were influenced by the low incidence of this finding (4/12-33\%) in our series. Gadolinium enhancement in brain lesions reflects disturbances of the blood-brain barrier (BBB) with high sensitivity. Disruption of the BBB is an early event in the development of inflammatory lesions in MS and a robust predictor of the occurrence of relapses ${ }^{29}$. Lebrun et al. ${ }^{5}$ demonstrated that patients whose baseline MRI scans exhibited Gd enhanced lesions had a substantially increased risk of developing new lesions. However, the authors emphasized that the risk of developing clinical symptoms was not defined.

Four of 12 (33.3\%) patients (2 males) developed an objective clinical symptom consistent with CNS inflammatorydemyelinating syndrome and fulfilled the criteria for either CIS or CDMS. The time taken for the development of clinical symptoms in our series ranged from 7 to 71 months (median time 2.9 years). Okuda et al. ${ }^{6}$ found a conversion rate of $22 \%$ (10/44) with a median time to clinical symptoms of 5.4 years, while Lebrun et al. ${ }^{5}$ found a conversion rate of 33\% (23/70) with a median time to CIS of 2.3 years. Both studies confirm that longer follow-up periods are crucial to defining MS conversion in many patients, but clinical conversion following RIS most commonly occurs during the initial years ${ }^{5,6}$.

In our series, all patients who converted to CDMS presented spinal cord and/or infratentorial lesions at the baseline MRI scan, and only one patient had a Gd enhanced lesion. A recent report regarding a large series of RIS patients confirmed that the presence of an affected spinal cord predicted the subsequent development of CIS or CDMS ${ }^{6}$. Bot et al. ${ }^{30}$ showed that spinal cord abnormalities are prevalent in early-stage MS, even in the absence of clinical manifestations. The authors confirmed that spinal cord lesions are useful to demonstrate DIS at the time of diagnosis ${ }^{30}$. Our study is in agreement with these previous reports; current data support the contention that spinal MRI should be performed in any patient with incidental demyelinating lesions in order to provide prognosis counseling ${ }^{27,30,31}$.

The concept of preclinical MS now challenges experts with several unanswered questions ${ }^{27}$. Standard neurologic evaluations often fail to detect cognitive impairments in MS patients, and some patients with RIS might also be cognitively impaired. Cognitive impairment was recently described to occur with similar frequency in early stage MS patients and RIS patients ${ }^{23}$. Brex et al..$^{32}$ postulated that the extent of inflammation and demyelination early in the course of MS may influence the extent of axonal loss later in the disease. The occurrence of a considerable number of lesions in RIS patients and the presence of black holes both allow us to identify RIS patients at risk of cognitive impairment, even from an initial examination. We propose that further clinical studies should address the role of the total number of lesions and black holes in the evolution of RIS in the cognitive performance of patients, even in the absence of symptoms.

We recognize several limitations of this study, mainly due to the number of patients and to the absence of neuropsychological testing. In our practice, very few patients without symptoms undergo spinal cord MRI evaluations, CSF analyses and visual evoked potential studies, thereby limiting a full analysis of this patient series. Similar studies have highlighted the importance of demonstrating DIS and DIT during the preclinical phase of lesions that are suggestive of demyelinating disease. Additional prospective studies are needed to determine the frequency of conversion of RIS to CDMS and to build strategies to manage RIS patients.

In conclusion, this Brazilian RIS cohort confirmed the predominance of female patients presenting with RIS. Most of the patients exhibited clinical or radiological progression in a short follow-up period. We propose that further studies are necessary to better define the risk of conversion in males and to clarify the role of the total number of brain lesions and black holes on cognitive function of RIS patients.

Our results provide evidence that infratentorial lesions, spinal cord involvement and the total number of lesions are more relevant than Gd enhancement in the prediction of both clinical and radiological progression. Although some individuals did not exhibit progression during a long follow-up period, we believe that all patients with RIS should be considered as having a high risk of developing MS. Infratentorial and spinal cord lesions are the best predictors for the inference of prognosis. We propose that a larger series of patients could shed more light on the natural course and evolution of incidental MRI lesions, and further assist in the management of patients in clinical practice. 


\section{References}

1. Okuda DT, Mowry EM, Beheshtian A, et al. Incidental MRI anomalies suggestive of multiple sclerosis: the radiologically isolated syndrome. Neurology 2009;72:800-805.

2. Siva A, Saip S, Altintas A, Jacob A, Keegan BM, Kantarci OH. Multiple sclerosis risk in radiologically uncovered asymptomatic possible inflammatory-demyelinating disease. Mult Scler 2009;15:918-927.

3. Sierra-Marcos A, Mitjana R, Castilló J, et al. [Demyelinating lesions as incidental findings in magnetic resonance imaging: a study of 11 cases with clinico-radiological follow-up and a review of the literature]. Rev Neurol 2010;51:129-134.

4. Lebrun C, Bensa C, Debouverie M, et al. Unexpected multiple sclerosis: follow-up of 30 patients with magnetic resonance imaging and clinical conversion profile. J Neurol Neurosurg Psychiatry 2008;79:195-198.

5. Lebrun C, Bensa C, Debouverie M, et al. Association between clinical conversion to multiple sclerosis in radiologically isolated syndrome and magnetic resonance imaging, cerebrospinal fluid, and visual evoked potential: Follow-up of 70 patients. Arch Neurol 2009;66:841-846.

6. Okuda DT, Mowry EM, Cree BA, et al. Asymptomatic spinal cord lesions predict disease progression in radiologically isolated syndrome. Neurology 2011;76:686-692.

7. Charil A, Yousry TA, Rovaris M, et al. MRI and the diagnosis of multiple sclerosis: expanding the concept of "no better explanation". Lancet Neurol 2006;5:841-852.

8. Poser CM, Paty DW, Scheinberg L, et al. New diagnostic criteria for multiple sclerosis: guidelines for research protocols. Ann Neurol 1983;13:227-231.

9. Barkhof F, Filippi M, Miller DH, et al. Comparison of MRI criteria at first presentation to predict conversion to clinically definite multiple sclerosis. Brain 1997;120:2059-2069.

10. Hawkes $\mathrm{CH}$, Macgregor AJ. Twin studies and the heritability of MS: a conclusion. Mult Scler 2009;15:661-667.

11. Phadke JG, Best PV. Atypical and clinically silent multiple sclerosis: a report of 12 cases discovered unexpectedly at necropsy. J Neurol Neurosurg Psychiatry 1983;46:414-420.

12. Lynch SG, Rose JW, Smoker W, Petajan JH. MRI in familial multiple sclerosis. Neurology 1990;40:900-903.

13. Tienari PJ, Salonen O, Wikström J, Valanne L, Palo J. Familial multiple sclerosis: MRI findings in clinically affected and unaffected siblings. J Neurol Neurosurg Psychiatry 1992;55:883-886.

14. de Seze J, Vermersch P. Sequential magnetic resonance imaging follow-up of multiple sclerosis before the clinical phase. Mult Scler 2005;11:395-397.

15. Lyoo IK, Seol HY, Byun HS, Renshaw PF. Unsuspected multiple sclerosis in patients with psychiatric disorders: a magnetic resonance imaging study. J Neuropsychiatry Clin Neurosci 1996;8:54-59.

16. Fisniku LK, Brex PA, Altmann DR, et al. Disability and T2 MRI lesions: a 20-year follow-up of patients with relapse onset of multiple sclerosis. Brain 2008;131:808-817.
17. Tintore M, Rovira A, Río J, et al. Baseline MRI predicts future attacks and disability in clinically isolated syndromes. Neurology 2006;67:968972.

18. Polman $\mathrm{CH}$, Reingold SC, Banwell B, et al. Diagnostic criteria for multiple sclerosis: 2010 revisions to the McDonald criteria. Ann Neurol 2011;69:292-302.

19. Swanton JK, Fernando K, Dalton CM, et al. Modification of MRI criteria for multiple sclerosis in patients with clinically isolated syndromes. $J$ Neurol Neurosurg Psychiatry 2006;77:830-833.

20. Swanton JK, Rovira A, Tintore M, et al. MRI criteria for multiple sclerosis in patients presenting with clinically isolated syndromes: a multicentre retrospective study. Lancet Neurol 2007;6:677-686.

21. Rovira A, Swanton J, Tintoré $M$, et al. A single, early magnetic resonance imaging study in the diagnosis of multiple sclerosis. Arch Neurol 2009;66:587-592.

22. Chataway J. When confronted by a patient with the radiologically isolated syndrome. Pract Neurol 2010;10:271-277.

23. Lebrun C, Blanc F, Brassat D, Zephir H, de Seze J. Cognitive function in radiologically isolated syndrome. Mult Scler 2010;16:919-925.

24. Brex PA, Miszkiel KA, O'Riordan JI, et al. Assessing the risk of early multiple sclerosis in patients with clinically isolated syndromes: the role of a follow up MRI. J Neurol Neurosurg Psychiatry 2001;70: 390-393

25. KortewegT,Tintoré M, Uitdehaag B, et al. MRI criteria for dissemination in space in patients with clinically isolated syndromes: a multicentre follow-up study. Lancet Neurol 2006;5:221-227.

26. Chard DT, Dalton CM, Swanton J, et al. MRI only conversion to multiple sclerosis following a clinically isolated syndrome.J Neurol Neurosurg Psychiatry 2011;82:176-179.

27. Bourdette $\mathrm{D}$, Yadav $\mathrm{V}$. The radiologically isolated syndrome revisited: when is it presymptomatic multiple sclerosis? Neurology 2011 76:680-681.

28. Bourdette D, Simon J. The radiologically isolated syndrome: is it very early multiple sclerosis? Neurology 2009;72:780-781.

29. Kappos L, Moeri D, Radue EW, et al. Predictive value of gadoliniumenhanced magnetic resonance imaging for relapse rate and changes in disability or impairment in multiple sclerosis: a meta-analysis. Gadolinium MRI meta-analysis group. Lancet 1999;353:964-969.

30. Bot JC, Barkhof F, Polman $\mathrm{CH}$, et al. Spinal cord abnormalities in recently diagnosed MS patients: added value of spinal MRI examination. Neurology 2004;62:226-233.

31. Polman $\mathrm{CH}$, Reingold SC, Edan G, et al. Diagnostic criteria for multiple sclerosis: 2005 revisions to the "McDonald criteria". Ann Neurol 2005;58:840-846.

32. Brex PA, Ciccarelli O, O’Riordan JI, Sailer M, Thompson AJ, Miller DH. A longitudinal study of abnormalities on MRI and disability from multiple sclerosis. N Engl J Med 2002;346:158-164. 